\title{
Competência profissional dos trabalhadores de programas de atividade física da atenção básica à saúde de Pernambuco
}

\author{
Professional competency of the professionals of physical activity programs of the \\ Pernambuco primary health care
}

\section{AUTORES \\ Danyelle de Cássia Ribeiro de Oliveira ${ }^{1}$ (D) Emmanuelly Correia de Lemos ${ }^{1}$ (D) \\ Caroline Ramos de Moura Silva² (D) Rafael Miranda Tassitano ${ }^{2,3}$ (D) \\ 1 Programa Academia da Cidade do Recife Secretaria Municipal de Saúde de Recife, Recife, Pernambuco, Brasil. \\ 2 Programa Associado de Pós-Graduação em Educação Física - UPE/UFPB, Recife, Pernambuco, Brasil. \\ 3 Departamento de Educação Física, Universidade} Federal Rural de Pernambuco, Recife, Brasil.

\section{CONTATO}

Rafael Miranda Tassitano rafael.tassitano@gmail.com

Rua Dom Manoel de Medeiros, S/N, Dois Irmãos, Recife,Pernambuco, Brasil. CEP: 52171-900.

DOI

$10.12820 /$ rbafs. $23 \mathrm{e} 0022$

\section{(a) $(1) \Theta$}

Copyright: This is an open-access article distributed under the terms of the Creative Commons Attribution License ${ }^{\circledR}$, which permits unrestricted use, distribution, and reproduction in any medium, provided that the original author and source are credited.

\begin{abstract}
RESUMO
O objetivo do estudo foi verificar a associação entre as competências profissionais de conhecimento, habilidade e atitude autopercebidas com a formação inicial, continuada, educação permanente e atuação profissional em programas de promoção da atividade física (PPAFs) na atenção básica à saúde de Pernambuco. Estudo transversal com 553 trabalhadores de 132 cidades. Competência profissional foi mensurada por 23 questões, com escala tipo likert nas dimensões de conhecimento $(n=7)$, habilidade $(n=9)$ e atitude $(n=7)$. Informações sociodemográficas, de tempo de atuação, formação inicial, continuada e educação permanente foram as variáveis independentes. $\mathrm{O}$ teste $k$-means não hierárquico foi utilizado para identificar grupos com maior e menor percepção de competência a partir do $Z$-scores do conhecimento, habilidade e atitude. Regressão logística binária bruta e ajustada foi utilizada para identificar os fatores associados a maior percepção de competência. Um total de $66,4 \%$ tinham formação em Educação Física, com menor quantidade de experiências durante a formação inicial e continuada. Ter formação superior ( $\mathrm{OR}=2,21$; IC95\%: 1,04-4,68), em Educação Física $(\mathrm{OR}=2,48$; IC95\%: 1,47-4,85), ser servidor público (OR = 1,86; IC95\%: 1,13-3,27) e participar de cursos, eventos e capacitações em atividade física no último ano ofertados pela gestão ( 1 - 2 cursos; $O R=3,86$; IC95\%: 2,17-6,81 e $\geq 3$ cursos; OR = 4,27; IC95\%: 1,99-7,58) aumenta a chance de percepção de maior competência. É necessário um redirecionamento na formação inicial, implementação de uma política de capacitação continuada com o foco na atenção básica de saúde, bem como a valorização do profissional fortalecido pelo tipo de vínculo.
\end{abstract}

Palavras-chave: Atenção básica à Saúde; Competência profissional; Atividade motora.

ABSTRACT

The objective of the study was to verify the association between professional competences in knowledge, ability and attitude with the initial, continuing education, permanent education and professional activity in physical activity promotion programs (PPAFs) of primary health care in Pernambuco. Cross-sectional study with 553 professionals from 132 cities. Professional competence was measured by 23 Likert questions in terms of knowledge $(n=7)$, ability $(n=9)$ and attitude $(n=7)$. Socio-demographic, actuation time, initial training, continuing education and permanent education were the independent variables. The non-bierarchical $k$-means test was used to identify groups with higher and lower perception of competence. Crude and adjusted binary logistic regression was used to identify the factors associated with higher perception of competence. About $66.4 \%$ have a Physical Education degree, with little experience in initial and continuing education. $(O R=2.21 ;$ CI95\%: 1.04-4.68) in Physical Education (OR $=2.48$; CI95\%: 1.47-4.85), to be a public servant $(O R=1.86 ; C I 95 \%: 1.13-3.27)$ and attend courses and physical activity training in last year (1 or 2 courses $-O R=3.86$; CI95\%: 2.17-6.81, and $\geq 3$ courses OR $=4.27 ;$ CI95\%: 1.99-7.58) increases the chance of perceived higher competence. It is necessary in the initial training, implementation of a policy of continuous training with the focus on basic health care, and valuation through the strengthening of the type of bond.

Keywords: Primary health care; Professional competency; Motor activity.

\section{Introdução}

A inserção do Profissional de Educação Física (PEF) no âmbito do Sistema Único de Saúde (SUS), ser anterior a implementação da Política Nacional de Promoção da Saúde (PNPS), que lançada em 2006, desencadeou diversas estratégias foram no território nacional, sendo uma dessas estabelecer como prioritária a promoção da prática de atividade física no âmbito do SUS ${ }^{1}$.

Para isso, foram publicados editais para fomento de Projetos e Programas de promoção da atividade física 
em mais de 1.500 municípios brasileiros ${ }^{2}$. Com isso, a inserção do PEF no SUS foi ampliada, sendo ainda mais potencializada após a criação do Núcleo de Apoio à Saúde da Família (NASF), em $2008^{3}$ e do Programa Academia da Saúde (PAS), em 20114.

Dessa forma, iniciaram-se debates entre profissionais, gestores e pesquisadores acerca da formação inicial e formação continuada, bem como, a atuação do PEF na atenção básica à saúde (ABS). Um dos focos é a necessidade de desenvolver competências comuns ou convergentes a várias profissões, uma vez que competências específicas não são suficientes para a atuação na perspectiva do SUS. Considera-se que as competências requeridas para atuação na $\mathrm{ABS}$ se constituem em ações para a consolidação do SUS, e que estas competências devem atender as necessidades de articulação da prática e educação, em uma perspectiva do cuidado à saúde ${ }^{5}$. A discussão sobre as competências dos profissionais de saúde é anterior em áreas como na Medicina, Odontologia, Enfermagem ${ }^{5,6}$ e mais recente na Educação Física ${ }^{7-11}$ Apesar de não existir um consenso quanto às competências do profissional para atuação na $A B S$, existem três eixos comuns: (a) conhecimentos, entendidos como o saber adquirido pelo profissional; (b) habilidades, como o saber fazer específico do profissional; e (c) atitudes, também entendidas como saber agir, julgar, escolher e decidir ${ }^{6}$.

Segundo Oliveira e colaboradores ${ }^{12}$ a maioria dos PEF que atuam na saúde pública não desenvolveram competências gerais e específicas para esta atuação, podendo justificar-se pela falta e/ou insuficientes experiências curriculares relacionadas ao contexto da ABS durante a graduação ${ }^{12}$. Programas de pós-graduações como, especializações, residências, mestrados profissionais na área de saúde coletiva e saúde da família passaram a ser opções de formação continuada, a fim de minimizar a carência da formação inicial. Assim como, de acordo com Política Nacional de Atenção Básica ${ }^{13}$, a educação permanente em saúde deve ser oportunizada pela gestão, a fim de emponderar os profissionais diante as novas exigências para atuação no serviço público de saúde.

Reconhecendo a importância de fomentar competências do PEF para atuação no âmbito do SUS, é necessário aprofundar investigações sobre o tema com uma abrangência municipal e estadual. Em face ao exposto, tem-se como objetivo verificar a associação entre as competências profissionais de conhecimentos, habilidades e atitudes autopercebidas com a formação inicial, continuada, educação permanente e atuação profissional em programas de promoção da atividade física (PPAFs) na ABS.

\section{Métodos}

Trata-se de um estudo transversal, com abrangência estadual, vinculado ao projeto intitulado "Avaliação dos programas e intervenções relacionadas à $\mathrm{AF}$ na rede de ABS no estado de Pernambuco - Projeto SUS+Ativo". O projeto foi submetido e aprovado pelo Comitê de Ética em Pesquisa com Seres Humanos da Universidade de Pernambuco - CAAE: 13373313.5.0000.5207. Informações mais detalhadas sobre concepção do projeto SUS+Ativo, construção e validação dos instrumentos e do método de campo foram publicadas por Barros et al. ${ }^{14}$.

Todos os programas de promoção da saúde da ABS que estavam vinculados às Secretarias de Saúde do Município ou da Secretaria de Saúde do Estado e que ofertavam ações sistemáticas de promoção da atividade física foram considerados elegíveis para o estudo. A população alvo a ser entrevistada foi constituída por todos trabalhadores que atuavam nos programas.

Para a coleta de dados foi utilizado um questionário previamente construído e validado e autoaplicado ${ }^{14}$, intitulado "Questionário para avaliação de intervenções para promoção da atividade física na atenção básica à saúde - versão profissional", constituída por três componentes, 16 dimensões e 151 perguntas. Para o presente estudo as dimensões utilizadas foram: sociodemográficas (sexo e idade); formação inicial (Se tem graduação superior, área da graduação, realizou estágio curricular obrigatório na $\mathrm{ABS}$, participação no programa PET-Saúde, participação de iniciação científica com temática na $\mathrm{ABS}$ ); atuação profissional (tempo de atuação na $\mathrm{ABS}$, tempo de atuação em programas de promoção da AF e tipo de vínculo), formação continuada (pós-graduação, residência multiprofissional) e educação permanente (quantidade de cursos, eventos ou capacitação nas diversas áreas de conhecimento nos últimos 12 meses oferecidos pela gestão e quantidade de cursos, eventos ou capacitações na área de atividade física nos últimos 12 meses oferecidas pela gestão); análise de competências (conhecimento, habilidade e atitude). A avaliação da competência profissional foi mensurada a partir 23 perguntas distribuídas entre os domínios percebidos de conhecimento $(n=7)$, habilidade $(n=9)$ e atitude $(\mathrm{n}=7)$, com opções de resposta em escala likert (muito baixo, baixo, médio, alto e muito alto).

A dimensão de conhecimento representa as aborda- 
gens e ferramentas para a promoção da atividade física na $\mathrm{ABS}$, enquanto que a dimensão de habilidade refere-se as ações de planejamento, comunicação, avaliação, incentivação e gestão, a fim de atender às demandas do local de atuação e por fim a dimensão de atitude esta ligada as atitudes e valores, tendo como exemplo, características pessoais que não estão, necessariamente, ligadas ao exercício específico de uma função. Os enunciados das questões e os complementos das mesmas por dimensão estão descritos no quadro a seguir:

O preenchimento do questionário ocorreu no local de trabalho e o tempo médio foi de 40 minutos. A participação foi voluntária e ocorreu após a assinatura do Termo de Consentimento Livre e Esclarecido. A tabulação dos dados foi realizada por meio da leitura ótica utilizando o software SPHYNX ${ }^{\circledast}$ (Sphynx Software Solutions Incorporation, Washington, Estados Unidos). Todas as análises foram realizadas utilizando o SPSS (versão 16.0).

No plano de análise dos dados foram realizadas aná- lises descritivas a partir da determinação da frequência (absoluta e relativa). Para as análises inferenciais todas as questões que compuseram as competências de conhecimento, habilidade e atitude foram somadas nas respectivas dimensões e posteriormente transformadas em Zscore. Esta padronização é um procedimento para a realização da análise de cluster que tem como objetivo a formação de padrões, isto é, um agrupamento de indivíduos em grupos de acordo a proximidade das respostas dadas nas variáveis de interesse. Para a análise de cluster utilizou-se o método não hierárquico $k$-means para a identificação de dois padrões considerando as dimensões de conhecimento, habilidade e atitudes.

Por fim, foi realizada regressão logística binária bruta e ajustada considerando o padrão identificado como mais positivo de percepção de competência profissional como desfecho e as variáveis sociodemográficas, formação inicial, atuação profissional, formação continuada e educação permanente foram consideradas as independentes. Para esta análise, algumas variáveis foram

Quadro 1 - Estrutura das questões por dimensão de competência profissional avaliada em trabalhadores de programas de atividade física da atenção básica à saúde de Pernambuco, 2016.

\begin{tabular}{|c|c|c|}
\hline Dimensão & Enunciado da questão & Complemento da questão \\
\hline Conhecimento & $\begin{array}{l}\text { Como o(a) senhor(a) classifica seu domínio } \\
\text { sobre promoção da atividade física no SUS, } \\
\text { mais especificamente sobre... }\end{array}$ & $\begin{array}{l}\text { abordagens de comunicação, informação e educação. } \\
\text { abordagens comportamentais e sociais. } \\
\text { monitoramento/avaliação das intervenções. } \\
\text { métodos e instrumentos para medida da atividade física. } \\
\text { ações em atividades físicas que acontecem na rede básica de saúde e na comunidade. } \\
\text { ações intersetoriais e de mobilização de parceiros para promoção da atividade física. } \\
\text { normas e regulamentos acerca da atividade física como estratégia de promoção da saúde } \\
\text { no SUS. }\end{array}$ \\
\hline Habilidade & $\begin{array}{l}\text { Como o(a) senhor(a) classifica o seu nível de } \\
\text { habilidade para... }\end{array}$ & $\begin{array}{l}\text { atuar em ações de planejamento de intervenções. } \\
\text { aconselhar usuários sobre a importância da prática de atividades físicas. } \\
\text { prescrever uma sessão de exercício físico para grupos específicos. } \\
\text { supervisionar grupos específicos que participam de atividade física. } \\
\text { realizar apoio matricial para profissionais de saúde sobre os benefícios da atividade } \\
\text { física. } \\
\text { desenvolver ações de promoção de atividades físicas articulado com os demais } \\
\text { profissionais de saúde. } \\
\text { desenvolver ações de promoção de atividade física de acordo com a realidade local. } \\
\text { dialogar com gestor local sobre questões que potencializem a realização de ações de } \\
\text { promoção da atividade física. }\end{array}$ \\
\hline Atitude & $\begin{array}{l}\text { Como o(a) senhor(a) classifica o nível em } \\
\text { que consegue demonstrar... }\end{array}$ & $\begin{array}{l}\text { autocontrole na realização de atividades em equipes multiprofissionais. } \\
\text { flexibilidade diante da complexidade e variabilidade da atuação profissional no contexto } \\
\text { da atenção básica à saúde. } \\
\text { criatividade diante da complexidade e variabilidade da atuação profissional no contexto } \\
\text { da atenção básica à saúde. } \\
\text { alteridade, colocando-se no lugar do outro, nas relações com os usuários e com os } \\
\text { demais profissionais de saúde. } \\
\text { consegue valorizar a importância das relações humanizadas nos serviços de saúde. } \\
\text { capaz de delegar tarefas e mobilizar outros profissionais em torno de projetos de } \\
\text { interesse na atenção básica à saúde. }\end{array}$ \\
\hline
\end{tabular}


categorizadas: faixa etária (20 - 29 anos; 30 - 39 anos e 40 anos ou mais); (tempo de atuação na $\mathrm{ABS}$ e tempo de atuação em programa de atividade física (até 1 ano, $>1$ e $\leq 3$ anos e $>3$ anos), tipo de vínculo (servidor público e outro tipo de vínculo), quantidade de cursos, eventos ou capacitações nas diversas áreas de conhecimento nos últimos 12 meses oferecidos pela gestão e quantidade de cursos, eventos ou capacitações na área de atividade física nos últimos 12 meses oferecidos pela gestão (nenhum curso, 1 a 2 cursos e 3 ou mais cursos). Em todos os testes inferenciais foi considerado um $\mathrm{p}<$ 0,05 para o nível de significância.

\section{Resultados}

Foram visitadas 132 cidades de todas as regiões do estado de Pernambuco que tinham programas com atividade física. Dois trabalhadores se recusaram participar e 10 estavam afastados (licença ou férias), participando efetivamente 553. Demais informações sociais, demográficas, relacionadas à formação inicial, continuada e de atuação profissional encontram-se na tabela 1 . Destaca-se que $7,1 \%$ relataram não ter formação superior.

Tabela 1 - Descrição das variáveis sociodemográficas, atuação profissional, relacionadas a formação inicial, continuada e educação permanente de trabalhadores que atuam em programas/intervenções de promoção da Saúde na atenção básica à saúde do estado de Pernambuco $(\mathrm{n}=553)$.

\begin{tabular}{lc}
\hline Variáveis & $\%(\mathrm{n})$ \\
\hline Sexo & $42,3(234)$ \\
Masculino & $57,7(319)$ \\
Feminino & \\
Faixa etária & $43,6(241)$ \\
Até 30 anos & $42,5(235)$ \\
31 - 40 anos & $13,9(77)$ \\
41 ou mais & \\
Graduado & $7,1(39)$ \\
Não & $92,9(514)$ \\
Sim & \\
Graduação em Educação Física & $33,6(186)$ \\
Não* & $66,4(367)$ \\
Sim & \\
Participou do PET Saúde & $92,6(512)$ \\
Não & $7,4(41)$ \\
Sim & \\
Estágio curricular obrigatório na ABS & $61,3(339)$ \\
Não & $38,7(214)$ \\
Sim & \\
Participou de IC na ABS & $86,3(477)$ \\
Não & $13,7(76)$ \\
Sim &
\end{tabular}

Continua...

\begin{tabular}{|c|c|}
\hline Variáveis & $\%(\mathrm{n})$ \\
\hline \multicolumn{2}{|l|}{ Tipo de vínculo } \\
\hline Sem vínculo formal & $2,0(11)$ \\
\hline Contrato temporário & $70,3(389)$ \\
\hline Servidor público & $26,8(148)$ \\
\hline Cargo comissionado & $0,9(5)$ \\
\hline \multicolumn{2}{|c|}{ Tempo de atuação na ABS } \\
\hline Até 1 ano & 25,1 (139) \\
\hline$>1$ ano até 3 anos & $38,9(215)$ \\
\hline 3 ou mais anos & $36,0(199)$ \\
\hline \multicolumn{2}{|c|}{ Tempo de atuação em PAF } \\
\hline Até 1 ano & $30,6(169)$ \\
\hline$>1$ ano até 3 anos & $36,3(201)$ \\
\hline 3 ou mais anos & $33,1(183)$ \\
\hline \multicolumn{2}{|l|}{ Pós-Graduação } \\
\hline Não & $44,1(244)$ \\
\hline Sim & $55,9(309)$ \\
\hline \multicolumn{2}{|c|}{ Residência multiprofissional } \\
\hline Não & $97,8(541)$ \\
\hline Sim & $2,2(12)$ \\
\hline \multicolumn{2}{|c|}{$\begin{array}{l}\text { Cursos, eventos ou capacitação nos últimos } 12 \text { meses } \\
\text { oferecidos pela gestão nas diversas áreas de conhecimento }\end{array}$} \\
\hline Nenhum & $15,7(87)$ \\
\hline $1-2$ cursos & $44,1(244)$ \\
\hline 3 ou mais & $40,1(222)$ \\
\hline \multicolumn{2}{|c|}{$\begin{array}{l}\text { Cursos, eventos ou capacitação na área da atividade } \\
\text { física nos últimos } 12 \text { meses oferecidos pela gestão }\end{array}$} \\
\hline Nenhum & $32,2(178)$ \\
\hline $1-2$ cursos & $37,4(207)$ \\
\hline 3 ou mais & $30,4(168)$ \\
\hline
\end{tabular}

* Assistente social (7,0\%), Farmácia (4,3\%), Enfermagem (1,6\%), Fisioterapia (40,9\%), Psicologia (13,4\%), Terapia Ocupacional (1,6\%), Nutrição (22,6\%), Fonoaudiologia (8,6\%); PET = Programa de Ensino Tutorial; $\mathrm{ABS}=$ atenção básica à saúde; $\mathrm{IC}=$ Iniciação científica; $\mathrm{PAF}=$ Programa de Atividade Física.

O cluster 1 agrupou 302 trabalhadores com os seguintes valores médios do Zscore de competência percebida para a dimensão conhecimento $(Z$ score $=0,596)$, habilidade $(Z$ score $=0,656)$ e atitude $(Z$ score $=0,557)$, nomeadamente de maior competência percebida. Já o cluster 2 agrupou 251 trabalhadores e os valores médios do Zscore observados foram: conhecimento ( $Z s^{-}$ core $=-0,695)$, habilidade $(Z$ score $=-0,770)$ e atitude $(Z$ score $=-0,639)$, nomeadamente o grupo que relatou menor competência percebida.

$\mathrm{Na}$ tabela 2 está apresentada as características e comparação entre os grupos identificados em relação as variáveis independentes do estudo. Em linhas gerais foram observadas diferenças significativas entre os grupos de maior e menor competência percebida para as variáveis de formação inicial (ter graduação e qual a área de formação); tempo de atuação (na ABS e no programa/intervenção de promoção da atividade física); formação continuada (pós-graduação) e educação 
permanente (cursos, eventos ou capacitação nas diversas áreas de conhecimento e na área da atividade física nos últimos 12 meses, oferecidos pela gestão).

Verificou-se uma maior proporção graduados, em Educação Física, com maior tempo de atuação tanto na $\mathrm{ABS}$ quanto no programa, que possui pós-graduação e que participaram de cursos, eventos e capacitações nas diversas áreas de conhecimento e na atividade física nos últimos 12 meses oferecidos pela gestão entre os trabalhadores do grupo de maior competência percebida quando comparado aos do grupo de menor competência percebida (Tabela 2).

Tabela 2 - Apresentação e comparação dos grupos identificados em relação as variáveis independentes dos profissionais que atuam em programas de promoção da saúde na atenção básica à saúde do estado de Pernambuco $(\mathrm{n}=553)$.

\begin{tabular}{|c|c|c|c|}
\hline Variáveis & $\begin{array}{c}\text { Maior competência } \\
\text { percebida } \\
\%(\mathrm{n})\end{array}$ & $\begin{array}{c}\text { Menor competência } \\
\text { percebida } \\
\%(\mathrm{n})\end{array}$ & $\mathrm{p}$ \\
\hline \multicolumn{3}{|l|}{ Sexo } & 0,013 \\
\hline Masculino & $51,7(156)$ & $31,1(78)$ & \\
\hline Feminino & $48,3(146)$ & $68,9(173)$ & \\
\hline \multicolumn{3}{|l|}{ Faixa etária } & 0,083 \\
\hline $18-30$ anos & $45,4(137)$ & $41,4(104)$ & \\
\hline $31-40$ anos & $43,7(132)$ & $41,0(103)$ & \\
\hline 41 ou mais & $10,9(33)$ & $17,5(44)$ & \\
\hline \multicolumn{3}{|l|}{ Graduado } & 0,022 \\
\hline Não* & $4,6(14)$ & $10,0(25)$ & \\
\hline Sim & $95,4(288)$ & $90,0(226)$ & \\
\hline \multicolumn{3}{|c|}{ Graduação em Educação Física } & 0,001 \\
\hline Não & $21,5(65)$ & $48,2(121)$ & \\
\hline Sim & $78,5(237)$ & $51,8(130)$ & \\
\hline \multicolumn{3}{|c|}{ Participou do PET Saúde } & 0,063 \\
\hline Não & $90,7(274)$ & $94,8(238)$ & \\
\hline Sim & $9,3(28)$ & $5,2(13)$ & \\
\hline \multicolumn{3}{|l|}{$\begin{array}{l}\text { Estágio curricular } \\
\text { obrigatório na } \mathrm{ABS}\end{array}$} & 0,493 \\
\hline Não & $62,6(189)$ & $59,8(150)$ & \\
\hline Sim & $37,4(113)$ & $40,2(101)$ & \\
\hline \multicolumn{3}{|l|}{$\begin{array}{l}\text { Participou de IC } \\
\text { na temática da } \mathrm{ABS}\end{array}$} & 0,107 \\
\hline Não & $84,1(254)$ & $88,8(223)$ & \\
\hline Sim & $15,9(48)$ & $11,2(28)$ & \\
\hline \multicolumn{3}{|l|}{ Tipo de vínculo } & 0,873 \\
\hline Outro & $73,5(222)$ & $72,9(183)$ & \\
\hline Servidor público & $26,5(80)$ & $27,1(68)$ & \\
\hline \multicolumn{3}{|c|}{ Tempo de atuação na ABS } & 0,032 \\
\hline Até 1 ano & $20,9(63)$ & $30,3(76)$ & \\
\hline$>1$ ano até 3 anos & 41,7 (126) & $35,5(89)$ & \\
\hline 3 ou mais anos & $37,4(113)$ & $34,3(86)$ & \\
\hline \multicolumn{3}{|c|}{ Tempo de atuação em PAF } & 0,012 \\
\hline Até 1 ano & $25,5(77)$ & $36,7(92)$ & \\
\hline$>1$ ano até 3 anos & $37,7(114)$ & $34,7(87)$ & \\
\hline 3 ou mais anos & $36,8(111)$ & $28,7(72)$ & \\
\hline
\end{tabular}

... continua

\begin{tabular}{|c|c|c|c|}
\hline Variáveis & $\begin{array}{c}\text { Maior competência } \\
\text { percebida } \\
\%(\mathrm{n}) \\
\end{array}$ & $\begin{array}{c}\text { Menor competência } \\
\text { percebida } \\
\%(\mathrm{n}) \\
\end{array}$ & $\mathrm{p}$ \\
\hline \multicolumn{3}{|l|}{ Pós-Graduação } & \multirow[t]{3}{*}{0,023} \\
\hline Não & $51,7(156)$ & $61,0(153)$ & \\
\hline $\operatorname{Sim}$ & $48,3(146)$ & $39,0(98)$ & \\
\hline \multicolumn{3}{|c|}{ Residência multiprofissional } & \multirow[t]{3}{*}{0,792} \\
\hline Não & $97,7(295)$ & $98,0(246)$ & \\
\hline Sim & $2,3(7)$ & $2,0(5)$ & \\
\hline \multicolumn{3}{|c|}{$\begin{array}{l}\text { Cursos, eventos ou capacitação } \\
\text { nos últimos } 12 \text { meses oferecidos } \\
\text { pela gestão nas diversas áreas } \\
\text { de conhecimento }\end{array}$} & \multirow[t]{4}{*}{0,013} \\
\hline Nenhum & $40,2(35)$ & $59,8(52)$ & \\
\hline $1-2$ cursos & $54,9(134)$ & $45,1(110)$ & \\
\hline 3 ou mais & $59,9(133)$ & $40,1(89)$ & \\
\hline \multicolumn{3}{|c|}{$\begin{array}{l}\text { Cursos, eventos ou capacitação } \\
\text { na área da atividade física nos } \\
\text { últimos } 12 \text { meses oferecidos } \\
\text { pela gestão }\end{array}$} & \multirow[t]{4}{*}{0,012} \\
\hline Nenhum & $28,7(51)$ & $71,3(127)$ & \\
\hline $1-2$ cursos & $66,2(137)$ & $33,8(70)$ & \\
\hline 3 ou mais & $67,9(114)$ & $32,1(54)$ & \\
\hline
\end{tabular}

* Assistente social (7,0\%), Farmácia (4,3\%), Enfermagem (1,6\%), Fisioterapia (40,9\%), Psicologia (13,4\%), Terapia Ocupacional (1,6\%), Nutrição (22,6\%), Fonoaudiologia (8,6\%); PET = Programa de Ensino Tutorial; $\mathrm{ABS}$ = atenção básica à saúde; $\mathrm{IC}=$ Iniciação científica; PAF $=$ Programa de Atividade Física.

A análise de regressão logística ajustada indicou que trabalhadores com formação superior e graduados em Educação Física apresentam maior chance de pertencer ao grupo que relataram maior competência profissional referida quando comparado àquele profissional sem formação superior e com formação superior fora da área de Educação Física. Outras duas variáveis foram significativas após a análise ajustada. Ser servidor público aumentou em quase duas vezes mais a chance de estar no grupo de maior competência profissional relatada. No entanto, a maior magnitude observada foi verificada entre aqueles que relataram ter participado de pelo menos 1 curso, evento ou capacitação na área da atividade física nos últimos 12 meses oferecidos pela gestão $(\mathrm{OR}=4,87$; IC95\%: 3,15-7,52), aumentado a chance quando a participação foi de 3 ou mais atividades dessa natureza $(\mathrm{OR}=5,25 ; \mathrm{IC} 95 \%: 3,32-8,31)$ (Tabela 3).

\section{Discussão}

Passado pouco mais de uma década desde a implementação da PNPS e do estabelecimento da atividade física como um eixo prioritário é possível verificar na literatura uma série de estudos sobre a universalização ao acesso, impacto na saúde do usuário, contudo pouco se tem investigado sobre as competências profissionais 
Tabela 3 - Análise de regressão bruta e ajustada tendo como desfecho a maior percepção de competência profissional e as demais variáveis de profissionais que atuam em programas de promoção da saúde na atenção básica à saúde do estado de Pernambuco $(\mathrm{n}=553)$.

\begin{tabular}{|c|c|c|c|c|c|c|}
\hline \multirow{2}{*}{ Variáveis } & \multicolumn{3}{|c|}{ Bruta } & \multicolumn{3}{|c|}{ Ajustada } \\
\hline & OR & IC95\% & $\mathrm{p}$ & OR & IC95\% & $\mathrm{p}$ \\
\hline \multicolumn{7}{|l|}{ Sexo } \\
\hline Masculino & 1 & & & 1 & & \\
\hline Feminino & 0,42 & $0,30-0,60$ & 0,001 & 0,68 & $0,50-1,03$ & 0,073 \\
\hline \multicolumn{7}{|l|}{ Faixa etária } \\
\hline $18-30$ anos & 1 & & & 1 & & \\
\hline $31-40$ anos & 0,97 & $0,67-1,40$ & 0,883 & 0,85 & $0,54-1,34$ & 0,483 \\
\hline 41 ou mais & 0,57 & $0,34-0,95$ & 0,033 & 0,60 & $0,32-1,10$ & 0,102 \\
\hline \multicolumn{7}{|l|}{ Graduado } \\
\hline Não* & 1 & & & 1 & & \\
\hline Sim & 2,28 & $1,17-4,48$ & 0,012 & 2,21 & $1,04-4,68$ & 0,033 \\
\hline \multicolumn{7}{|c|}{ Graduação em Educação Física } \\
\hline Não & 1 & & & & & \\
\hline Sim & 2,98 & $2,34-4,91$ & 0,001 & 2,48 & $1,47-4,85$ & 0,001 \\
\hline \multicolumn{7}{|c|}{ Participou do PET-Saúde } \\
\hline \multicolumn{7}{|l|}{ Não } \\
\hline Sim & 1,87 & $0,94-3,69$ & 0,074 & 1,08 & $0,49-2,42$ & 0,387 \\
\hline \multicolumn{7}{|c|}{ Estágio curricular obrigatório na $\mathrm{ABS}$} \\
\hline Não & 1 & & & 1 & & \\
\hline Sim & 0,88 & $0,63-1,25$ & 0,493 & 0,98 & $0,63-1,56$ & 0,905 \\
\hline \multicolumn{7}{|l|}{ Iniciação científica } \\
\hline Não & 1 & & & 1 & & \\
\hline $\operatorname{Sim}$ & 1,50 & $0,91-2,47$ & 0,110 & 1,40 & $0,78-2,53$ & 0,255 \\
\hline \multicolumn{7}{|l|}{ Vínculo profissional } \\
\hline Outro & 1 & & & 1 & & \\
\hline Servidor público & 1,03 & $0,70-1,50$ & 0,872 & 1,96 & $1,13-3,27$ & 0,022 \\
\hline \multicolumn{7}{|c|}{ Tempo de atuação na ABS } \\
\hline Até 1 ano & 1 & & & 1 & & \\
\hline$>1$ ano até 3 anos & 1,70 & $1,11-2,62$ & 0,021 & 1,77 & $0,93-3,35$ & 0,082 \\
\hline$>3$ anos & 1,58 & $1,02-2,45$ & 0,046 & 1,78 & $0,89-3,58$ & 0,108 \\
\hline \multicolumn{7}{|c|}{ Tempo de atuação com AF } \\
\hline Até 1 ano & 1 & & & 1 & & \\
\hline$>1$ ano até 3 anos & 1,56 & $1,03-2,36$ & 0,038 & 1,23 & $0,62-2,42$ & 0,542 \\
\hline$>3$ anos & 1,84 & $1,20-2,81$ & 0,010 & 1,09 & $0,63-2,43$ & 0,834 \\
\hline \multicolumn{7}{|l|}{ Pós-Graduação } \\
\hline Não & 1 & & & 1 & & \\
\hline Sim & 1,46 & $1,04-2,05$ & 0,032 & 1,25 & $0,82-1,86$ & 0,313 \\
\hline \multicolumn{7}{|c|}{ Residência multiprofissional } \\
\hline Não & 1 & & & 1 & & \\
\hline $\operatorname{Sim}$ & 1,16 & $0,46-2,25$ & 0,803 & 1,26 & $0,35-4,58$ & 0,711 \\
\hline \multicolumn{7}{|c|}{$\begin{array}{l}\text { Cursos, eventos ou capacitação nos últimos } 12 \text { meses } \\
\text { oferecidos pela gestão nas diversas áreas de conhecimento }\end{array}$} \\
\hline Nenhum & 1 & & & 1 & & \\
\hline $1-2$ cursos & 1,81 & $1,10-2,97$ & 0,018 & 1,22 & $0,71-2,43$ & 0,272 \\
\hline 3 ou mais & 2,22 & $1,34-3,68$ & 0,021 & 1,75 & $0,95-2,92$ & 0,082 \\
\hline \multicolumn{7}{|c|}{$\begin{array}{l}\text { Cursos, eventos ou capacitação na área da atividade física } \\
\text { nos últimos } 12 \text { meses oferecidos pela gestão }\end{array}$} \\
\hline Nenhum & 1 & & & 1 & & \\
\hline $1-2$ cursos & 4,87 & $3,15-7,52$ & 0,001 & 3,86 & $2,17-6,81$ & 0,001 \\
\hline 3 ou mais & 5,25 & $3,32-8,31$ & 0,001 & 4,27 & $1,99-7,58$ & 0,001 \\
\hline
\end{tabular}

*Assistente social, Farmácia, Enfermagem, Fisioterapia, Psicologia, Terapia Ocupacional, Nutrição, Fonoaudiologia. 
para a atuação. Os principais resultados deste estudo indicaram que trabalhadores dos programas de promoção da atividade física da ABS de Pernambuco com formação superior, sobretudo em Educação Física, servidores públicos e que participaram de cursos, eventos e capacitações na área da atividade física nos últimos 12 meses oferecidos pela gestão relataram uma maior autopercepção de competência profissional quando comparado aos demais.

A construção da percepção de competência profissional é um processo complexo e temporal, estando relacionada com a formação inicial vinculada a um contexto de aprendizagem formal e ao processo de formação continuada e de educação permanente. Em adição esta percepção também é influenciada pelos processos de aprendizagem informal e pelo ambiente organizacional do campo de atuação e pela própria prática profissional. Segundo Camelo et al. ${ }^{6}$, o conhecimento entendido como o saber adquirido, as habilidades que remete o saber fazer específico e as atitudes entendida como o saber agir, julgar, escolher e decidir constituem os três eixos da competência profissional. Para este estudo a dimensão de conhecimento teve como foco as abordagens e ferramentas para atuação, a dimensão de habilidade nas ações de planejamento, comunicação, avaliação, incentivação e gestão e a dimensão de atitude relacionada às atitudes e valores.

Considerando todos os profissionais, cerca de seis em cada dez são formados em Educação Física. Independentemente do grupo ao qual o profissional pertenceu em relação à competência percebida foi verificado uma pequena proporção dos que tiveram experiências relacionadas à $\mathrm{ABS}$ na formação inicial, não havendo diferença significativa entre os grupos. Apesar de pequena, foi verificada ainda uma proporção de 7,1\% de trabalhadores que atuavam como profissionais, mas sem formação superior.

A literatura tem indicado que a competência na atuação profissional depende tanto da utilização adequada de conhecimentos e procedimentos quanto a domínios inerentes ao saber específico da formação ${ }^{5,6}$. Embora a formação em Educação Física ainda permaneça de forma distanciada das demandas dos serviços públicos de saúde ${ }^{10}$, com o novo mercado de trabalho os profissionais passaram a ter que adquirir novos conhecimentos e habilidades para um melhor desempenho ${ }^{9}$, podendo justificar tais achados.

Apesar de o PEF apresentar as competências específicas para orientar ações da área de conhecimento, alguns autores também defendem que profissionais de outras categorias de saúde devem trabalhar no cuidado da população, através das ações de aconselhamento, orientação e encaminhamento para programas de atividade física $^{15,16}$. A portaria $\mathrm{n}^{\mathrm{o}} 2.681$, que redefiniu o PAS, aponta que além dos profissionais de Educação Física as atividades do PAS também serão desenvolvidas por outros profissionais da $\mathrm{ABS}$, inclusive aqueles que atuam na Estratégia Saúde da Família e nos NAFS, quando houver ${ }^{17}$. Estes podem contribuir para o empoderamento da população acerca do autocuidado, tornando-se agentes multiplicadores. Esses dados podem justificar a presença de profissionais de outras formações em saúde atuando na promoção da atividade física. Porém, a maioria desses profissionais não possui conhecimento no campo de atividade física, como em relação às recomendações globais para promoção da atividade física promoção ${ }^{11}$, destacando a necessidade de espaços de formação para demais profissionais da saúde sobre promoção da $\mathrm{AF}$ na $\mathrm{ABS}$.

Percebe-se uma desarticulação entre as políticas da saúde e da educação que reflete no distanciamento entre a formação profissional e as necessidades do sistema de saúde. Apesar do SUS ser uma das oportunidades de mercado de trabalho na área da saúde, o investimento das universidades em uma formação que atenda as exigências profissionais para atuação de qualidade no serviço público ainda é incipiente ${ }^{18}$. Nesse sentido, não se pode perder de vista a reforma curricular que atenda as expectativas das áreas da saúde que se propõe a atuar na saúde pública. Maior presença de disciplinas como epidemiologia, organização e gestão do SUS, sociologia, ABS, saúde pública e estágios dentro da estrutura do SUS, além de um número maior de cursos de pós-graduação ou estágios multiprofissionais na saúde pública com a presença de estudantes e docentes da Educação Física ${ }^{19}$.

Como também, as vivências práticas nos serviços do $\mathrm{SUS}^{20}$, ou especificamente na ABS, são escassas ou inexistente ${ }^{21}$ entre as disciplinas ofertadas do eixo da saúde. Logo, as instituições de ensino superior necessitam repensar as estruturas curriculares, tendo em vista a ampliação dos campos de atuação profissional, e as mudanças ocorridas no sistema de saúde vigente.

$\mathrm{Na}$ tentativa de minimizar estes problemas, alguns autores discutem a reorientação do processo de formação inicial mais contextualizado, estimulando a interdisciplinaridade e o multiprofissional, além de uma educação permanente para aproximar o ensino ao 
sistema de saúde e qualificar o profissional, através de capacitações ou cursos e pós-graduações ${ }^{21,22}$.

Destacam-se algum as iniciativas interministeriais que têm buscado reorientar a formação profissional, quanto à estruturação curricular e troca de saber, para atender as necessidades de saúde do Brasil. O Programa Nacional de Reorientação da Formação Profissional em Saúde (Pró-Saúde), voltado inicialmente para os cursos da Medicina, Enfermagem e Odontologia ${ }^{23}$, tem agregado os demais cursos da área da saúde por meio do Programa de Educação para o Trabalho em Saúde (PET-Saúde $)^{24}$. A oportunidade de aproximar o ensino e o serviço promove vivências práticas aos discentes que contribuirão efetivamente na qualificação profissional e melhor assistência às necessidades da população.

$\mathrm{Na}$ formação complementar, o Ministério da Saúde tem contribuído na expansão das Residências Multiprofissionais em Saúde da Família, como estratégia de desenvolvimento de competências profissionais, para a renovação da força de trabalho em saúde ${ }^{25}$. Porém, essas estratégias parecem não atingir a maioria desses profissionais, destacando os resultados encontrados no presente estudo, em que a maioria dos profissionais não teve essas experiências durante a formação.

Quanto ao tipo de vínculo, destaca-se que no grupo de maior percepção existe uma maior proporção de profissionais servidores. Importante destacar que a maior parte destes trabalhadores são do Programa Academia da Cidade do Recife que é um programa institucionalizado e o ser o único no estado de Pernambuco que proporciona estabilidade profissional. Por ser um programa institucionalizado e com dezesseis anos de atuação, garante espaços como Encontros de Educação Permanente uma vez por mês, o que possibilita emponderar os profissionais quanto a sua atuação no âmbito da ABS. Por outro lado, sabe-se que a maioria dos vínculos profissionais no âmbito da saúde pública nos programas é por contrato temporário ${ }^{26}$, sem processo de seleção, o que gera a possibilidade de indicação do recurso humano sem uma formação adequada, principalmente em municípios considerados de pequeno porte. Essa fragilidade pode ser encontrada tanto em trabalhadores sem formação superior, como também em profissionais com nível superior completo. Portanto, a precarização do vínculo profissional na ABS é um dos principais problemas a serem superados para a qualificação do cuidado em saúde ${ }^{12,27}$.

Outro aspecto gira em torno da necessidade em incentivar a reorientação do modelo de formação das profissões da saúde de acordo com as necessidades assistenciais, pactuando com os gestores do SUS, um trabalho em articulação com os pólos de capacitação na $\mathrm{ABS}^{17}$ Assim como a experiência prévia de atuação na perspectiva da saúde coletiva pode influenciar positivamente na humanização do serviço, demonstrando a importância da realização de um treinamento introdutório para início do trabalho das equipes de saúde ${ }^{28}$.

As pontuações citadas acima estão de acordo com Política Nacional de Educação Permanente em Saúde, que apresenta a Educação Permanente como aprendizagem no trabalho, onde o aprender e o ensinar se incorporam ao cotidiano das organizações e ao trabalho. A educação permanente se baseia na aprendizagem significativa e na possibilidade de transformar as práticas profissionais ${ }^{29}$. Esta deve ser realizada de forma sistemática, visto a amplitude de conhecimentos as serem compartilhados entre os profissionais para uma atuação de qualidade, como também pela alta rotatividade de profissionais no SUS.

A contribuição deste estudo destaca-se principalmente por apresentar a relação de componentes da formação e da atuação com uma maior ou menor percepção de competência para o trabalho da Promoção de atividade física na ABS. Corroborando, pontos já investigados por outros pesquisadores, como: (a) a necessidade de uma formação inicial e continuada alinhada à atuação do PEF na ABS; (b) profissionais de saúde em geral, formados para dentro da sua atuação generalista, promover a $\mathrm{AF}$ de forma qualificada; (c) minimização dos vínculos de profissionais preconizados e (d) o estabelecimento de rotina de educação permanente no âmbito dos serviços da $\mathrm{ABS}$.

Para avançar o campo de conhecimento sugerem-se estudos que investiguem de que forma as competências estão sendo trabalhadas na formação inicial nos cursos de graduação, bem como relatar realidades exitosas nesse processo de formação. No presente não foram encontradas diferenças significativas entre os grupos de maior e menor percepção de competência no que tange ter tido a experiência na graduação (Iniciação científica, estágio, participação do PET-Saúde). Contudo os autores acreditam ser de suma importância expandir nos currículos e (re)discutir estas experiências no processo formal de ensino. Sugere-se ainda o mapeamento de ações e experiências de formação continuada e de educação permanente que são realizadas por intermédio dos municípios e estados como ferramenta no processo de trabalho, verificando os limites em torno do conteúdo promoção da atividade física com relação aos 
princípios e diretrizes da $\mathrm{ABS}$.

Entre as limitações destacamos que a utilização de um único tipo de instrumento (questionário) não permitiu aprofundar elementos importantes relacionados a construção da competência profissional, principalmente da formação inicial (por exemplo: acesso ao currículo e ementas do curso de formação inicial) educação continuada, bem como o teor dos cursos e ações apoiadas oferecidas pela gestão. Sugere-se a incorporação de análise documental junto a gestão, entrevista semi-estruturada com profissionais para potencializar e aprofundar essa discussão.

No campo de atuação, equipes multidisciplinares podem contribuir de forma a superar a fragmentação do conhecimento, gerando uma troca de saberes entre especialistas de diferentes grupos profissionais ${ }^{30}$. Isto pode levar ao empoderamento das demais categorias a fim de que estes se tornem agentes multiplicadores quanto a promoção da atividade física. Porém é necessário que o Profissional de Educação Física saiba quais as competências a serem desempenhadas na equipe.

\section{Conflito de interesses}

Os autores declaram não haver conflito de interesses.

\section{Financiamento}

Fundação de Amparo a Ciência e Tecnologia de Pernambuco (FACEPE). Número: APQ-0287-4.09/13. Conselho Nacional de Ciência e Tecnologia (CNPQ). Número: APQ 485911/2013-2

\section{Contribuição dos autores}

Oliveira DCR, participou da concepção do projeto, redação do artigo e revisão crítica relevante do conteúdo intelectual. Tassitano RM, participou da concepção do projeto, responsabilizou-se pela análise dos dados, revisão crítica do artigo e aprovação da versão a ser publicada. Lemos EC e Silva CRM, atuaram na revisão crítica do artigo e aprovação da versão a ser publicada.

\section{Agradecimento}

Agradecemos a todos que contribuíram de maneira direta e indiretamente para concretização deste Projeto, em especial aos usuários, profissionais, gestores, ao Grupo de Pesquisa em Estilo de Vida e Saúde e ao órgão financiador (CNPQ).

\section{Referências}

1. Departamento de Análises de Situação em Saúde, Secretaria de Vigilância à Saúde, Secretaria de Atenção à Saúde. Política Nacional de Promoção da Saúde: PNPS: revisão da Portaria MS/GM no 637, de 30 de março de 2006. Brasilia: Ministério da Saúde; 2014. Disponível em: http://bvsms.saude.gov.br/bvs/ publicacoes/pnps_revisao_portaria_687.pdf.Acesso em 16/01/18.
2. Malta D, Silva MMA, Albuquerque GM, Amorim RCAR, Araújo GB, Silva TS, et al. Política Nacional de Promoção da Saúde, descrição da implementação do eixo atividade física e práticas corporais, 2006 a 2014. Rev Bras Ativ Fís Saúde. 2014;19(3):286-99.

3. Ministério da Saúde. Núcleo de Apoio da Saúde da Família. Portaria Ministerial de Saúde, nº154, de 24 de janeiro de 2008.

4. Brasil. Ministério da Saúde. Portaria GM/MS n. 719, de 07 de abril de 2011. Institui o programa academia da saúde no âmbito do Sistema Único de Saúde. Diário Oficial da União.

5. Castro EAB, Campos SEM. Uma discussão sobre as competências e habilidades de gestores no âmbito do sistema público de saúde. Revista APS. 2003;6(2):94-8.

6. Camelo SHH, Angerami ELS. Competência profissional: a construção de conceitos, estratégias desenvolvidas pelos serviços de saúde e Implicações para a enfermagem. Texto Contexto Enferm. 2013;22(2):552-60.

7. Coutinho SS. Competências do profissional de Educação Física na atenção básica à saúde [Tese de doutorado]. São Paulo. Escola de Enfermagem de Ribeirão Preto; 2011.

8. Feitosa WMN, Nascimento JV. As competências específicas do profissional de Educação Física que atua na orientação de atividades físicas: um estudo Delphi. Rev Bras Ciên Mov. 2003;11(4):19-26.

9. Nascimento JV. Escala de auto-percepção de competência profissional em educação física e desportos. Rev Paul de Educ Fís. 1998;13(1):5-21.

10. Falci DM, Belisário AS. A inserção do profissional de educação física na atenção primária à saúde e os desafios em sua formação. Interface (Botucatu). 2013;17:885-99.

11. Florindo AA, Andrade DR, Guerra PH, Mota J, Crone D, Mafra ACCN, et al. Physical activity promotion by health practitioners: a distance-learning training component to improve knowledge and counseling. Prim Health Care Res Dev. 2017;10:1-11.

12. Oliveira DCR, Brito ALS, Tassitano RM. Perfil dos profissionais que atuam nos programas de promoção da saúde com intervenções em atividade física da atenção básica à saúde do estado de Pernambuco. Rev Bras Ativ Fís Saúde. 2016;21(5):442-51.

13. Departamento de Atenção Básica, Secretaria de Atenção à Saúde, Ministério da Saúde. Política Nacional de Atenção Básica. Brasília: Ministério da Saúde, 2012. Disponível em: http://189.28.128.100/dab/docs/publicacoes/geral/pnab.pdf. Acesso em 16/01/2018.

14. Barros MVG, Lemos EC, Silva JRA, Moura CR, Fonseca SA, Tassitano RM. Avaliação dos programas e intervenções de promoção da atividade física na atenção básica à saúde de Pernambuco: construção, validação de instrumentos e método de trabalho de campo do Projeto SUS+Ativo. Rev Bras Ativ Fís Saúde. 2016;21(5):388-99.

15. Jacobson DM, Strohecker L, Compton MT, Katz DL. Physical activity counseling in the adult primary care settings. Am J Prev Med. 2005;29(2):158-62.

16. Orrow G, Kinmonth AL, Sanderson S, Sutton S. Effectiveness of physical activity promotion based in primary care: systematic review and meta-analysis of randomised controlled trials. BMJ. 2012;344:e1389.

17. Brasil. Ministério da Saúde. Portaria n. 2,681, de 07 de novembro de 2013. Redefine o programa academia da saúde no âmbito do Sistema Único de Saúde. Diário Oficial da União. 
18. Campos FE, Ferreira JS, Feuerwerker L, Sena RR, Campos JJB, Cordeiro H, et al. Caminhos para aproximar a formação de profissionais de saúde das necessidades da atenção básica. Rev Bras Educ Méd. 2001;25:53-9.

19. Salvador EP. Propagando a Atividade Física. Rev Corpoconsciência. 2014;18:1-7.

20. Rosa FK. Currículo de Educação Física das Universidades Públicas do Sul do Brasil: enfoque no Sistema Único de Saúde [Monografia]. Graduação em Educação Física. Universidade Federal de Santa Catarina; 2011.

21. Brugnerotto FA. Caracterização dos currículos de formação profissional em Educação Física: um enfoque sobre saúde [Dissertação de Mestrado]. Piracicaba (SP): Universidade Metodista de Piracicaba; 2008.

22. Tavares MFL, Rocha RM, Bittar CML, Petersen CB, Andrade M. A promoção da saúde no ensino profissional: desafios na saúde e a necessidade de alcançar outros setores. Ciênc Saúde Colet. 2016;21(6):1799-808.

23. Brasil. Portaria Interministerial no 3.101 , de 03 de novembro de 2005. Institui o Programa de Bolsas para a Educação pelo Trabalho e cria a Comissão Nacional de Residência Multiprofissional em Saúde - CNRMS. Diário Oficial da União 2004; 4 nov.

24. Brasil. Portaria Interministerial no 1.802 , de 26 de agosto de 2008. Institui o Programa de Educação pelo Trabalho para a Saúde - PET-Saúde. Diário Oficial da União 2008; 26 ago.

25. Nascimento DDG, Oliveira MAC. Reflexões sobre as competências profissionais para o processo de trabalho nos Núcleos de Apoio à Saúde da Família. O Mundo da Saúde. 2010;34(1):92-6.
26. Rodrigues JD, Ferreira DKS, Silva PA, Caminha IO. Inserção e atuação do profissional de educação física na atenção básica à saúde: revisão sistemática. Rev Bras Ativ Fís Saúde. 2013;18(1):5-15.

27. Santos SFS. Núcleo de Apoio à Saúde da Família no Brasil e a atuação do profissional de Educação Física [Dissertação de Mestrado]. Florianópolis (SC): Universidade Federal de Santa Catarina; 2012.

28. Trad LAB, Rocha AARM. Condições e processo de trabalho no cotidiano do Programa Saúde da Família: coerência com princípios da humanização em saúde. Ciênc Saúde Colet. 2011;16(3):1969-80.

29. Departamento de Gestão da Educação em Saúde, Secretaria de Gestão do Trabalho e da Educação na Saúde. Política Nacional de Educação Permanente em Saúde. Brasília: Ministério da Saúde; 2009. Disponível em: http://bvsms. saude.gov.br/bvs/publicacoes/politica_nacional_educacao_ permanente_saude.pdf. Acesso em 16/01/2018.

30. Minelli DS, Soriano JB, Fávaro PE. O profissional de Educação Física e a intervenção em equipes multiprofissionais. Rev Movimento. 2009;15(04):35-62.

Recebido: $14 / 05 / 2018$

Aprovado: 03/09/2018

\section{Como citar este artigo:}

Oliveira DCR, Lemos EC, Silva CRM, Tassitano RM. Competência profissional dos trabalhadores de programas de atividade física da atenção básica à saúde de Pernambuco. Rev Bras Ativ Fís Saúde. 2018.23:e0022. DOI: 10.12820/rbafs.23e0022 